\title{
Virulence of the plant-associated endophytic fungus Lecanicillium muscarium to diamondback moth larvae
}

\author{
Michal Kuchár ${ }^{*}$, Travis R. Glare ${ }^{1}$, John G. Hampton¹, Ian A. Dickie ${ }^{2}$ and Mary C. Christey ${ }^{3}$ \\ ${ }^{1}$ Bio-Protection Research Centre, PO Box 85084, Lincoln University 7647, New Zealand \\ ${ }^{2}$ Bio-Protection Research Centre, School of Biological Sciences, University of Canterbury, \\ Christchurch 8140, New Zealand \\ ${ }^{3}$ The New Zealand Institute of Plant and Food Research, Private Bag 4704, Christchurch 8140, \\ New Zealand \\ ${ }^{*}$ Corresponding author: michal.kuchar@lincolnuni.ac.nz
}

\begin{abstract}
Plutella xylostella (diamondback moth) is a prominent pest of brassicas which is now resistant to most insecticides. Despite years of research, the range of available products used in biological control of diamondback moth is still somewhat limited. We isolated putative endophytic fungi from New Zealand cabbage plants to search for unique biological control agents of diamondback moth larvae. The larvae were fed leaf discs from commercially grown cabbage covered in spores from endophytic fungal isolates to test the insecticidal properties of these fungi. Twenty of the 52 fungal isolates tested failed to kill any diamondback moth larvae. However, three isolates of Lecanicillium muscarium induced mortality greater than $80 \%$. While these isolates have potential for use in biological control applications, further research into propagation, formulation, and method, rate and timing of application is needed.
\end{abstract}

Keywords Endophytes, biological control, entomopathogenic fungi

\section{INTRODUCTION}

Plutella xylostella (Lepidoptera, Plutellidae) is commonly known as the diamondback moth (DBM) and is a prominent pest of Brassica species. It attacks not only vegetables such as cabbages, but also forage brassicas and canola (Brassica napus) (Furlong et al. 2013). Worldwide, production of vegetable Brassica species is estimated to contribute US $\$ 26$ billion to the global economy. However, the cost of DBM control combined with crop yield losses can reach US $\$ 2.7$ billion annually (Furlong et al. 2013).

Control of DBM is currently achieved mostly through the use of broad-spectrum synthetic insecticides. However, indiscriminate use of these chemicals has led to insecticide resistance in various populations of DBM. There are more than 80 compounds falling into all major classes of insecticides for which resistance in
DBM has been recorded (Furlong et al. 2013). Insecticide application is increasingly regarded as unsustainable and may be associated with many health and environmental risks (Gilden et al. 2010; Lukowicz et al. 2018). Broadspectrum insecticides may also be harmful to other invertebrates, which could include natural predators of DBM, thereby reducing the environmental capacity for controlling DBM populations (Furlong et al. 2013).

Diamondback moth has a wide range of natural enemies, including viruses, fungi, bacteria, generalist predators and parasitoids. Parasitic wasps can attack larvae or pupae of DBM. The most commonly promoted species are wasps of the genera Diadegma, Diadromus or Cotesia, since some can reduce DBM populations by up to $80 \%$ (Gurr et al. 2018; Hermansson 2016). Use of the bacterium Bacillus thuringiensis 
(Bt) has proven to be very effective in controlling DBM in many parts of the world. However, DBM was the first insect to develop field resistance to a Bt toxin, probably due to indiscriminate use, similar to the resistance developed to synthetic insecticides (Furlong et al. 2013).

Diamondback moth is susceptible to several genera of entomopathogenic fungi. Fungal genera such as Lecanicillium, Isaria or Metarhizium have shown ability to control DBM populations. However, the most widely used fungus in field conditions is Beauveria bassiana (Gurr et al. 2018).

Biological control of DBM is a promising tool to tackle the damage caused by this insect, especially because of possible synergistic effects with additional biological control agents. Synergy in DBM control was achieved when using Beauveria bassiana and $\mathrm{Bt}$ toxins or predators attacking different larval stages (Gurr et al. 2018). Farmers are increasingly looking for environmentally friendly methods to control insect pests. Use of biological control agents is considered less toxic to humans and the environment than chemical pesticides (Brimner \& Boland 2003; Heydari \& Pessarakli 2010).

One source of new agents to control pests of brassica crops may be organisms already present in brassica plants. Fungal and bacterial endophytes can have beneficial properties, including inducing pest resistance in the plant (Card et al. 2015). As part of a larger study on endophytes of Brassica species in New Zealand, we tested 52 isolates from 35 species of fungi obtained from cabbage plants (Brassica oleracea var. capitata) to determine if any had direct virulence against DBM larvae.

\section{MATERIALS AND METHODS}

Diamondback moth larvae were obtained from a colony maintained for 10 years at Lincoln University, New Zealand. The colony was established in 2008 with DBM collected at fields at Lincoln University, New Zealand. The colony consists of twelve separate populations that are routinely mixed between each other, in addition to wild DBM individuals to prevent loss of genetic diversity. Resistance to insecticides or Btbased biopesticides has not been observed in the colony. For bioassays, second-instar DBM larvae were fed leaf discs from commercially grown cabbage plants that were covered in spores from selected fungal isolates to test the insecticidal properties of these fungi.

\section{Experimental treatment}

Putative endophytic fungi were isolated from cabbage plants in 2015 and 2016, following procedures described in a study by Zhang et al. (2014). Selected fungal isolates were grown for 3-4 weeks on 1/4 strength potato-dextrose agar (PDA; Difco, BD, USA) in a growth chamber at $18^{\circ} \mathrm{C}$, with $12 \mathrm{~h} / 12 \mathrm{~h}$ - light/dark period. Conidia of each isolate were harvested from the plates into sterile aqueous $0.01 \%$ Triton X-100 (Sigma-Aldrich, New Zealand), which was then filtered through sterile cotton wool. Conidial concentration was adjusted to approximately $10^{7}$ conidia/mL. A disc was cut from the cabbage leaf using a $30-\mathrm{mm}$ cork borer. Leaf discs were washed thoroughly in sterile distilled water and dried using tissue paper prior to treatment. A filter paper disc (30-mm diameter) was placed in a $35-\mathrm{mL}$ clear graduated plastic portion cup and moistened with $100 \mu \mathrm{L}$ of sterile distilled water. Fifty-microlitre aliquots of each conidial suspension were deposited on each side of the leaf disc and spread over the surface using an inoculation loop so that the whole disc area was evenly covered. Treated leaf discs were allowed to air dry for about 30 minutes before putting them on top of the moist filter paper disc. Five secondinstar DBM were placed in each cup with a leaf disc using a fine camel brush. The cup was closed and placed in an incubator at $25^{\circ} \mathrm{C}$, with $12 \mathrm{~h} / 12 \mathrm{~h}$ - light/dark period. There were six bioassays in total, each testing a subset of the 52 isolates. All isolates were tested once, except Lecanicillium muscarium isolate m2La6, which was tested in two separate bioassays. Each bioassay was arranged in a complete randomised design with 4 replicates. 


\section{Measurements}

Larval mortality was assessed daily for at least four days after inoculation by observation using a stereo microscope. Unless in a cocoon, live larvae were feeding and moving around the leaf disc and responded to even a slight shake or when touched by a brush. Larvae were considered dead when they did not respond to physical stimuli, and when brown discolouration suggested decomposition of larval tissues (Fig. 1).

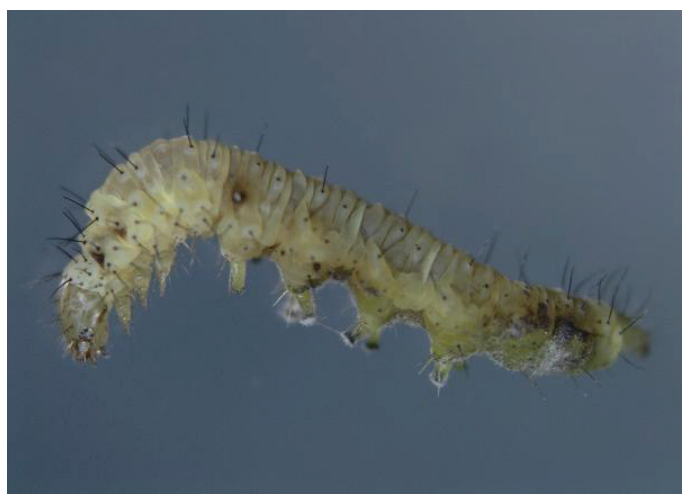

Figure 1 A dead Plutella xylostella larva, showing brown discolouration of internal tissues.

\section{Statistical analyses}

Mean mortalities were compared four days after inoculation in each bioassay using an ANOVA test and subsequent Tukey's honest significant difference test in the $\mathrm{R}$ environment ( $\mathrm{R}$ Core Team 2016).

\section{RESULTS}

Twenty of the 52 isolates tested failed to kill any DBM larvae (Fig. 2). However, three isolates of Lecanicillium muscarium induced mortality greater than $80 \%$ and two other isolates induced mortality just under $50 \%$. Isolate $223 \mathrm{~S} 3512$ caused the highest DBM mortality of $90 \%$ (Tukey HSD $\mathrm{P}>0.001$ ), while strains a2R14 and 223S352 both killed $81 \%$ of the larvae (Tukey HSD P>0.001). In one experiment, L. muscarium isolate m2La6 caused $48 \%$ mortality, which was significantly different from the control treatment (Tukey HSD $\mathrm{P}=0.02$ ), but in a repeated experiment the mortality was $43 \%$ and the effect was not significant in a post-hoc analysis. $L$. muscarium isolate h2La53 induced 25\% mortality, which statistically did not differ from the control. Results are summarised in Figure 2.

\section{DISCUSSION}

This study found that three of six isolates of the fungus L. muscarium (all isolated from cabbage tissues) increased mortality of DBM larvae in a feeding bioassay. The pathogenicity of the $L$. muscarium isolates used in this study is similar to results of other studies which reported the potential of Lecanicillium species as a biological control agent of DBM. However, the Lecanicillium spp. isolates used in biopesticide formulations have rarely targeted DBM; usually the controlled pests have been aphids or nematodes (Goettel et al. 2008). A review by Brodeur (2012) summarised several commercial products based on Lecanicillium species, including products aimed at controlling aphids, mites, thrips and/ or whiteflies. A number of studies have reported showing potential for biological control of DBM. In general, the effects of Lecanicillium species were consistently better than no treatment, but were usually poorer than the most effective treatments, either a synthetic insecticide or another entomopathogenic fungus. For example, Yamada et al. (2009) found that the strains of hybrid Lecanicillium species used in their study at a concentration of $10^{6}$ conidia $/ \mathrm{mL}$ killed half of the DBM larvae after just two days and resulted in more than $80 \%$ mortality in a full screening bioassay, but these isolates were less effective under field conditions even at concentration of $2 \times 10^{7}$ conidia $/ \mathrm{mL}$. More recently, Duarte et al. (2016) found that at a concentration of $10^{7}$ conidia/mL a L. muscarium isolate caused more than $80 \%$ mortality of DBM larvae feeding on cabbage leaf discs, while isolates of Beauveria bassiana and Metarhizium rileyi caused 98-100\% mortality. In the current study, DBM mortality ranged from $25 \%$ to $90 \%$ after 4 days as a result of contact with the L. muscarium conidia. This variability in pathogenicity could be attributed to different virulence strategies among isolates as described by Bye and Charnley (2008), who 
Diamondback moth larvae mortality

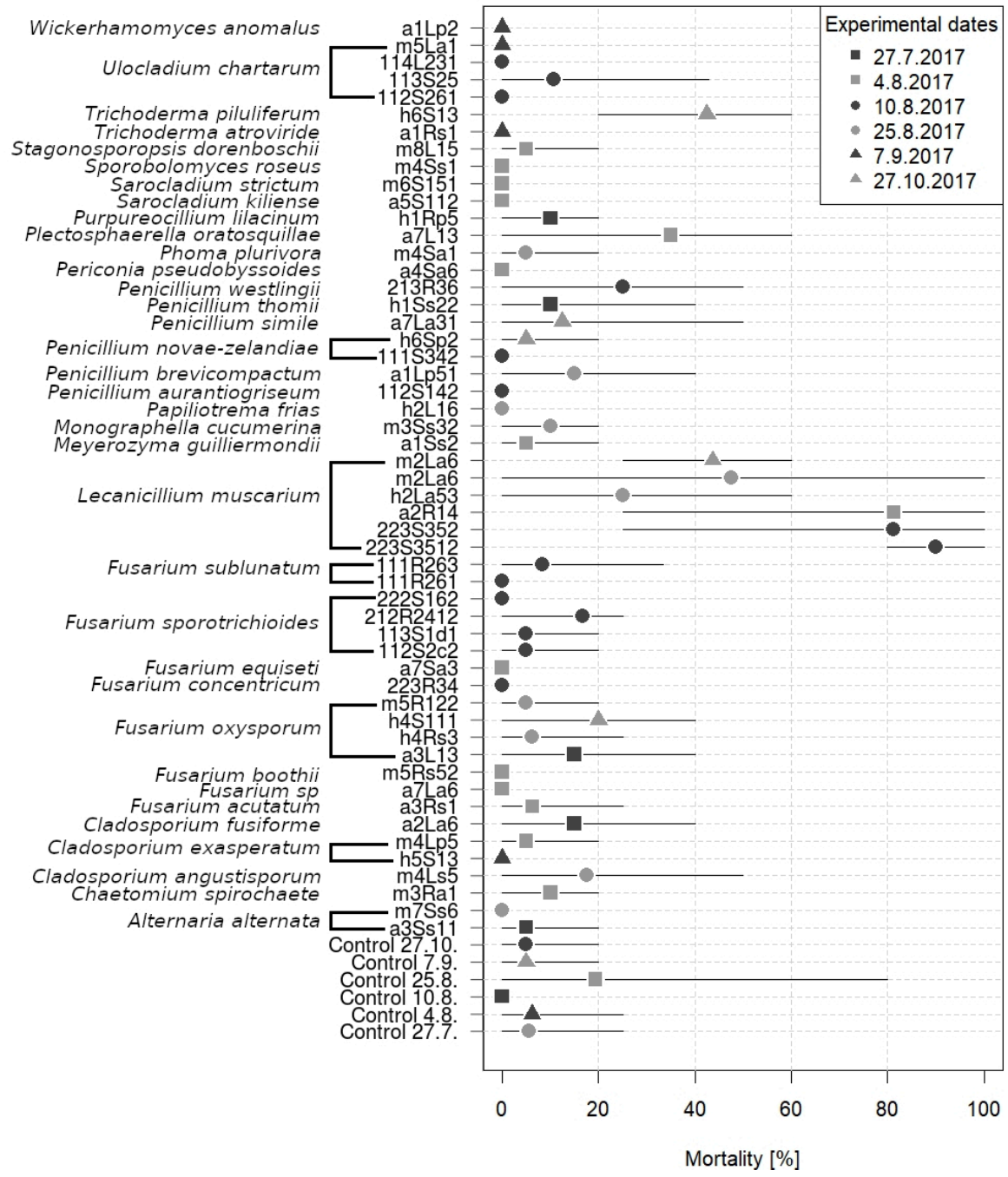

Figure 2 Mortality of Plutella xylostella larvae following treatment with a range of fungal isolates. Symbols represent mean mortality (\%) in respective bioassays. Whiskers represent the range of observed values.

showed that various isolates of Lecanicillium species possessed a distinctly expressed battery of cuticle-degrading enzymes. Similar to the cuticle degrading enzymes, other metabolites involved in virulence may have differential expression, such as a recently described bassianolide which suppresses the immune response of DBM (Keppanan et al. 2018).

The current study evaluated only the effect of contact of DBM larvae with conidia already on the surface of cabbage leaves, but there is evidence that endophytic fungi can influence insect feeding and development. For example, Acremonium alternatum, a fungal endophyte, reduced DBM feeding and growth rate when applied to the roots of Brussels sprouts (Brassica oleracea var. gemmifera) (Raps \& Vidal 1998). Similarly, endophytic Metarhizium anisopliae increased 
mortality of DBM larvae feeding on oilseed rape (Brassica napus) (Batta 2013). Furthermore, the presence of endophytic Lecanicillium lecanii in the leaves of cotton plants slowed reproduction rates of aphids (Gurulingappa et al. 2010). Since isolates in this study originated from internal plant tissues, there is a possibility they are able to endophytically colonise plant tissues and could affect feeding and reproductive patterns or even mortality of DBM. In the current study, potent isolates of L. muscarium were obtained from stems $(223$ S3512, 223S352) and roots (a2R14) of cabbage plants, while an isolate recovered from leaf tissue caused lower mortality of DBM. Interestingly, the L. lecanii isolate used to control aphids in a study by Gurulingappa et al. (2010) originated from the leaves of cotton plants. However, the tissue preference of the isolates that had the highest mortality of DBM in the current study, or their potential for DBM control when endophytic is not yet known. The effect of an endophytic entomopathogen is usually less substantial compared to direct contact of DBM with conidia (Batta 2013). Nevertheless, research on the extent and nature of the interactions between the fungus and host plant could improve the biological control of DBM, where the reduced effect on mortality could be traded for reduced costs bypassing the need to apply conidia with regards to the presence of susceptible pest stages. However, both approaches would require further research into propagation, formulation, dosage and application manner and timing (Lacey et al. 2001).

Expanding laboratory experiments to field trials is often problematic so other key steps include establishing the practicalities and cost effectiveness of scaling up microorganism production and determining the efficacy of fungal biological control agents under field conditions. The current study evaluated the effect of only a single concentration of conidial suspension on DBM mortality hence a dose response experiment should be one of the first steps in further research, as if the required dose is too high, a product would not be economically viable. Mass production of Lecanicillium spp. conidia have been achieved by either solid substrate or submerged liquid fermentation (Jaronski 2014). Potential inundative or endophytic use of $L$. muscarium isolates from current study would define appropriate production method and formulation. Laboratory experiments usually result in higher virulence than those under field conditions (Yamada et al. 2009) so field trials are necessary to assess the efficacy of biological control agent used compared with available commercial products. Also, it is critical to test the interactions of fungal biological control agents with possible non-target species to ensure there is no loss of ecosystem services (Brimner \& Boland 2003; Roy \& Pell 2000) before these biological control agents could be considered for use in large-scale field applications. In addition, testing for human safety is imperative if the isolates are to be used with crops for human consumption, such as cabbage.

\section{CONCLUSIONS}

Of 52 fungal isolates recovered from cabbage tissues, only three showed potential as biological control agents of DBM. All three active isolates were strains of L. muscarium. However, this study needs to be followed by further research if the active fungal isolates obtained here are to be developed as biological control agents for field conditions. Mechanisms underlying the observed variability of effects, dose response, temporal dynamics of the induced mortality as well as endophytic properties and interactions with other organisms of these fungal isolates should be further investigated.

\section{ACKNOWLEDGEMENTS}

We would like to acknowledge the work of Jenny Brookes and Josefina Narciso from the BioProtection Research centre for maintaining DBM colonies and assisting with establishment of the bioassays. We would also like to thank the New Zealand Ministry of Business, Innovation and Employment, Grasslanz Technology Ltd and the Foundation for Arable Research for their funding of the Next Generation Biopesticides Programme of which this project is a part. 


\section{REFERENCES}

Batta YA 2013. Efficacy of endophytic and applied Metarhizium anisopliae (Metch.) Sorokin (Ascomycota: Hypocreales) against larvae of Plutella xylostella L. (Yponomeutidae: Lepidoptera) infesting Brassica napus plants. Crop Protection 44: 128-134.

Brimner T, Boland GJ 2003. A review of the nontarget effects of fungi used to biologically control plant diseases. Agriculture, Ecosystems and Environment 100: 3-16.

Brodeur J 2012. Host specificity in biological control: insights from opportunistic pathogens. Evolutionary Applications 5: 470480.

Bye NJ, Charnley AK 2008. Regulation of cuticledegrading subtilisin proteases from the entomopathogenic fungi Lecanicillium spp: implications for host specificity. Archives of Microbiology 189: 81-92.

Card SD, Hume DE. Roodi D, McGill CR, Millner JP, Johnson RD 2015. Beneficial endophytic microorganisms of Brassica - A review. Biological Control 90: 102-112.

Duarte RT, Gonçalves KC, Espinosa DJL, Moreira LF, De Bortoli AS, Humber RA, Polanczyk RA 2016. Potential of entomopathogenic fungi as biological control agents of Diamondback moth (Lepidoptera: Plutellidae) and compatibility with chemical insecticides. Journal of Economic Entomology 8(38): 275-301.

Furlong, MJ, Wright DJ, Dosdall LM 2013. Diamondback moth ecology and management: Problems, progress, and prospects. Annual Review of Entomology 58: 517-541.

Gilden RC, Huffling K, Sattler B 2010. Pesticides and health risks. Journal of Obstetric, Gynecologic, \& Neonatal Nursing 39(1): 103-110.

Goettel MS, Koike M, Kim JJ, Aiuchi D, Shinya R, Brodeur J 2008. Potential of Lecanicillium spp. for management of insects, nematodes and plant diseases. Journal of Invertebrate Pathology 98: 256-261.

Gurr, GM, Reynolds, OL, Johnson, AC,
Desneux, N, Zalucki, MP, Furlong MJ, Li Z, Akutse KS, Chen J, Gao X, and You M 2018. Landscape ecology and expanding range of biocontrol agent taxa enhance prospects for diamondback moth management. A review. Agronomy for Sustainable Development 38(3), e23 https://doi.org/10.1007/s13593018-0500-z.

Gurulingappa P, Sword GA, Murdoch G, McGee PA 2010. Colonization of crop plants by fungal entomopathogens and their effects on two insect pests when in planta. Biological Control 55(1): 34-41.

Hermansson J 2016. Biology of the Diamondback moth (Plutella xylostella) and its future impact in Swedish oilseed rape production. Technical report. Swedish University of Agricultural Sciences, Uppsala, Sweden, 42 p. http://stud.episilon.slu.se

Heydari A, Pessarakli M 2010. A review on biological control of fungal plant pathogens using microbial antagonists. Journal of Biological Sciences 4: 273-290.

Jaronski ST 2014. Chapter 11 - Mass Production of Entomopathogenic Fungi: State of the Art, In: Morales-Ramos JA, Rojas MG, ShapiroIlan DI Eds. Mass Production of Beneficial Organisms. Academic Press, Pp. 357-413.

Keppanan R, Sivaperumal S, Mubasher H, Dash CK, Bamisile BS, Qasim M, Wang L 2018. Investigation and molecular docking studies of Bassianolide from Lecanicillium lecanii against Plutella xylostella (Lepidoptera: Plutellidae). Comparative Biochemistry and Physiology 206/207: 65-72.

Lacey LA, Frutos R, Kaya HK, Vail P 2001. Insect pathogens as biological control agents: do they have a future? Biological Control 21(3): 230-248.

Lukowicz C, Ellero-Simatos S, Régnier M, Polizzi A, Lasserre F, Montagner A, Lippi Y, Jamin EL, Martin J-F, Naylies C, Canlet C, Debrauwer L, Bertrand-Michel J, Al Saati T, Théodorou V, Loiseau N, Mselli-Lakhal L, Guillou H, Gamet-Payrastre L 2018. Metabolic effects of a chronic dietary exposure to a low-dose pesticide cocktail in mice: sexual dimorphism 
and role of the constitutive androstane. Environmental Health Perspectives 126(6), CID 067007.

RCore Team 2016. R: A language and environment for statistical computing. R Foundation for Statistical Computing, Vienna, Austria.

Raps A, Vidal S 1998. Indirect effects of an unspecialized endophytic fungus on specialized plant-herbivorous insect interactions. Oecologia 114: 541-547.

Roy HE, Pell JK 2000. Interactions between entomopathogenic fungi and other natural enemies: implications for biological control. Biocontrol Science and Technology 10: 737752.

Yamada K, Aiuchi D, Koike M, Masuda T 2009. Possibility of microbial control using entomopathogenic fungi Lecanicillium spp. hybrid strains and Beauveria bassiana against the diamondback moth. IOBC-WPRS Bulletin 45: 331-334.

Zhang Q, Zhang J, Yang L, Zhang L, Jiang D, Chen W, Li G 2014. Diversity and biocontrol potential of endophytic fungi in Brassica napus. Biological Control 72: 98-108. 